\title{
Tadeusz Czarnecki
}

\section{G.E. MOORE ON LOGICAL POSSIBILITY}

Aristotle interprets modalities in a manner consistent with common sense. Necessity, possibility and contingency are modes in which sentences are related to their properties of being true or false. Accordingly, modal sentences concern the actual world. As a sentence becomes true in virtue of its relation to the world, the sentence should firstly be materially true if one is to determine whether it is necessarily or contingently true. A sentence is true if it describes as existing what actually exists and simply cannot be true if its content does not refer to the actual world.

Truth is primarily understood as actual truth, whereas possible truth means actual falsehood: a sentence is possibly true only if it is actually false. Actual truth is then in contradiction with possible truth: what is actually true cannot be at the same time actually false, i.e., possibly true. When truth is consistently interpreted in connection with the actual world, one interprets a necessary truth as a sentence that is actually true and could not have been false: never was or will be false.

Logic introduces compound sentences that are necessarily true in virtue of they form, i.e., the role connectives or quantifiers play in them. One of the most celebrated is the law of contradiction: $p$ and not- $p$ cannot both be true. Yet formal logic often lacks tools to decide whether a conjunction including two contradictory sentences, especially modified by operators, is necessarily false. One sometimes needs philosophical arguments in order to decide that a conjunction including two modified contradictory sentences is necessarily false.

Logic suggests that actuality has noting to do with explaining modalities. There are two basic inter-definable modal notions: necessity and possibility, 
and it is preferable to concentrate on necessity, the more complicated one. As possibility is related to necessity, it is enough to explain necessity to understand what is meant by possibility. Basic questions seem to be: (1) Which truths are necessary? and (2) How can necessary truths be characterized? They are troublesome because necessary truths do not create a homogeneous class. It is therefore hard to imagine that one can propose a theory of necessity that would cover all different cases.

Logicians accentuate that necessity means for them only logical necessity: necessary truths are those whose denials are self-inconsistent. Indisputable examples are to be the truths of logic, mathematics and analytic truths, characterized as sentences in which the concept of the subject contains the concept of the predicate. None of them fulfill the Aristotelian criterion of being material truths. The former ones are explicitly called formal truths; the latter are qualified as non-substantial, linguistic truths. Since they do not concern the actual world, it is difficult to say that they are actually true. It is dubious whether logicians believe that they will discover necessary truths among material truths.

Their disbelief in material necessary truths is discernible in the way they distinguish logical necessity from pseudo-necessity. They acknowledge that philosophy provides various explanations of necessity but all are unsatisfactory: sentences proposed to be necessary do not fulfill the criterion of describing facts that could not have been otherwise. ${ }^{1}$

1. Conditional inevitablility. A fact is sometimes called necessary when it is a necessary result or correlate of some antecendent conditions. These conditions are themselves such exceptional facts that it is beyond people's power to prevent another fact, if they are fulfilled. The result or correlate is then meant to be necessary because it is an inevitable one. This explanation is unsatisfactory since it proposes a conditional notion of necessity. Even if the result actually follows or co-exists, it is imaginable that the antecendent conditions themselves are not inevitable, could have been prevented, or the relationship between these conditions and their result could have changed, i.e., they would cease to entail their result, if some further, more general assumptions would not be satisfied.

2. Inalterability of the past. A fact that had already happened in the past is called necessary in the sense that it is irreversible and inalterable. Of every fact one has the right to say that if it had happened in the closed

\footnotetext{
${ }^{1}$ Konyndyk, K. (1986). Introductory Modal Logic, Notre Dame: University of Notre Dame, ss. 12-14.
} 
past and is detached from the present, it is impossible to be erased, changed or prevented. Before it happened it was possible to prevent it but after it happened it is now impossible to remove or change it. People can build their future but cannot rebuild their past.

For logicians, a sentence describing a past fact is clearly not necessary insofar as the fact itself was contingent, i.e., it need not have happened. Though the past is inalterable, it is logically open in the sense that there was a time when the past facts could have been prevented: before they happened, they need not have happened. The past is inalterable from the viewpoint of the present but it was still wide open before it once became the present. Logicians are dissatisfied with the necessity of past facts because they are in a sense only semi-necessary. Though all sentences which were true descriptions of facts are now true and will be true, on condition that they are sufficiently unequivocal, logicians complain that when they became originally true, they were accidentally true.

3. Non-temporal physical necessity. The actual world is thought to be governed by laws, which seem to be non-temporal, i.e., unchangeable in time. The laws were, are and will be the same. As non-temporally valid, they seem to be fully necessary. Yet they are under suspicion because logicians are convinced that the world itself is contingent in every respect. Although physical laws seem unchangeable in the world, they could have been different if the world changed. As far as the world preserves its identity, the laws perhaps cannot be different. Nevertheless, the world itself could have changed into another world, and the laws could, too. The identity of the world is contingent. It follows that the laws of physics, which do not determine but only express its identity, are also contingent. Physical laws, inviolable in this very world, do not have to remain valid in another world. If the world as an ultimate whole is accidental, its essential qualities are accidental, too.

Logically necessary truths are supposed to exceed pseudo-necessary truths mentioned above because the latter, even if true in a strong sense, could have been false, whereas logically necessary truths are to be sentences whose denials are self-contradictory, i.e., they are not only true but could not have been false. Despite many favorable appearances, the latter must be classified as truths of a worse kind: logically possible truths, i.e., sentences whose denials are not self-contradictory. In this way the term "logically possible truth" spreads over all sentences that are not logically necessary, including also actually true ones. Somewhat unexpectedly, actually false and true sentences are thrown into the same category and become similar. It is a strange result because two contradictory sentences $p$ and not- $p$ seem to create 
a consistent pair just because they belong to the same category. When one says ' $p$ and not-p', one utters a contradiction. Nevertheless, when one asserts a conjunction of two possible truths 'It is possible that $p$ and it is possible that not-p', then the conjunction as a whole ceases to be a contradiction: it should be interpreted as a possible truth.

The concept of necessity belonged for ages to the domain of epistemology. And the basic pair of opposite concepts was not necessity and possibility but necessity and contingency. The problem of necessity was expressed in the question: What can be known as absolutely true? Usually, as far as philosophers believed in knowledge transcending sensual phenomena, they believed that there are necessary truths. Logicians, neglecting the legacy of epistemology, claim that epistemic conceptions of necessity are unsatisfactory and should be carefully distinguished from the logical conception of necessity. They find it important to criticize epistemic criteria of necessity; to make it clear that no epistemic notion of necessity separately or all notions summarily are equivalent to the notion of logical necessity. ${ }^{2}$

1. Ungiveupable. There are beliefs that are psychologically ungiveupable but they have nothing to do with logical necessity. For example, some firstperson present-tense assertions are ungiveapable, i.e., it is unimaginable that a person who makes them would be prone to withdraw them in any circumstances. The assertion 'I exist' is one of them. But everybody can see that its third-person version ' $A$ exists' is unquestionably a contingent sentence, i.e., it is possible to be false. Being perhaps pragmatically ungiveapable, 'I exist' is clearly only contingently true.

2. Unable to be rationally rejected. Some sentences are such that no argument against them is more convincing than they themselves. They are acceptable merely in virtue of the fact that they are asserted. Cartesian famous assertion 'I am thinking' can be rationally rejected only on the condition that it is not uttered meaningfully. But again the third-person statement ' $A$ is thinking' clearly is not a necessary truth: it can be false. More generally, inability to reject an assertion is mainly a psychological matter and people are unable to reject false sentences because they lack sufficient knowledge. Moreover, from the fact that a sentence is a necessary truth it does not follow that it cannot be rationally rejected, as it happens, for example, when an ignorant wrongly thinks that a deceiver is trying to fool him while asserting in fact a necessary truth.

\footnotetext{
${ }^{2}$ Plantinga, A.(1974). The Nature of Necessity, Oxford: Clarendon Press, ss. 1-9.
} 
3. Self-evident. Logicians complain that the notion of self-evidence does not exclude all contingent truths. Some unquestionable necessary truths are self-evident, yet some of them are not. Still worse, there are contingent truths that seem to be self-evident. For example, Moore repeatedly proposes lists of claims of common sense that are epistemically symmetrical: nobody either doubts them or needs justification to accept them. Wittgenstein develops this idea and holds that Moore's sentences describe the necessary conditions of language functioning. According to him, the language actually used would collapse if people began to doubt their identity, the existence of the world or unchangeability of some properties of the world.

4. Known a priori . If a priori refers to the truths that are known prior to sense experience, it is obvious that there are necessary truths that are simply not known. Logicians rarely express their opinions on whether there are also necessary truths that are known a posteriori . Anyway, the category of truths known a priori seems too narrow, as there are necessary truths that are not known. Proponents of the view that some sentences known a posteriori are necessary say that these sentences describe indispensable qualities of objects, i.e., an object $X$ could not be identified as $X$, if $X$ lost its indispensable quality $Y$.

Logicians call epistemic explanations of necessity pseudo-explanations. They think that the notion of necessity is irreducible to epistemic notions. The concept of logical necessity is for them an ontological one, i.e. not relative either to anyone's actual knowledge or ability to know.

The ontologization of modal concepts is typically made in two steps. The correspondence theory of truth is expanded and modal operators are interpreted as quantifiers. In order to expand the theory of truth logicians introduce possible worlds and say that a sentence is possibly true if it relates to a possible fact in a possible world. The operator of possibility becomes a counterpart of the existential quantifier: it refers to a possible world; the operator of necessity becomes a counterpart of the universal quantifier: it refers to every possible world. It is obvious that logicians are forced to ontologize if they decide to impose the quantificational interpretation on modal concepts. They are also used to considering meaningfulness of modal sentences in terms of expanded truth-conditions: a sentence is meaningless only if it is false in all possible worlds.

They do not mind that this explanation is circular as it presupposes that one already understands the notion of logical possibility when one theorizes about possible worlds in order to explain the notion of possible truth. They are happy that thanks to possible worlds the basic explanation of the notion 
of logical necessity becomes simple and universal. A sentence is called a necessary truth if it is true in all possible worlds A sentence is a possible truth if it is true in a possible world; it does not matter much whether it is at the same time actually false or true. A sentence is contingent if it is both a possible truth and possible falsehood.

The notion of contingent truth is still reserved to the actual world. A sentence is a contingent truth if it is actually true but not necessarily true. Other definitions of true sentences have somewhat unnatural consequences for anyone who believes that the problem to solve is whether there is anything necessary in the world. Suddenly, the problem ceases to concern the actual world. The actual world loses its exceptional position and becomes a world. A necessary truth ceases to be a specific truth of any particular world; it does not characterize the actual world. Actual truths are reduced to a sub-class of possible truth. Accordingly, the actual world becomes just one of possible worlds.

David Lewis, a radical proponent of modal realism, maintains that possible worlds are not mere descriptions - they contain things that are as real as actual things. ${ }^{3}$ The actual world is just one of many that exist. Modal realism is for him an ontological thesis with no epistemological implications. It does not imply that people know that possible worlds exist because the worlds are totally isolated from each other and from people who actually exist.

To distinguish possible worlds from the actual one, Lewis suggests that the word "actual" should be construed as an indexical term. The meaning of the word is established by the speaker's uttering it: if a person $A$ uses the expression "actual world", it refers to the world in which $A$ exists. The word "actual" resembles the word "I": as "I" does not refer to a particular person but always to the person $A$ who utters it, so "actual" does not refer to a particular world but to the one in which the expression "actual world" is uttered by $A$. No particular world is the actual one in itself. It is a world $A$ exists in that is the actual one for $A$. This explanation has an interesting consequence: since a speaker $A$ exists only in one world and he is a part of the world, all true first-person present-tense assertions made by $A$ about himself are restricted only to the actual world, i.e., they are false in all possible worlds. Treating the word "actual" as an indexical term, Lewis opens the possibility that sentences including the indexical term "I" acquire specific qualities.

Modal realism faces various difficulties. Lewis claims that all possible worlds exist necessarily, whereas one can argue that they exist accidentally. If a speaker $A$ is a logician who believes that the actual world is contin-

${ }^{3}$ Chihara, C. (1998). The Worlds of Possibility, Oxford: Clarendon Press, ss. 76-112. 
gent and accidental, i.e., it could have been different or even need not have existed, then the existence of a possible world should be for him equally contingent and accidental. If so, it is difficult to admit that the explanation of the operator of necessity as a quantifier ranging over possible worlds is satisfactory. If people have no criterion to decide whether a sentence is a necessary truth, when the sentence concerns the actual world, and are sceptical whether any sentence could have been necessary because the world is contingent as a whole, then the question is why a sentence that is true in all possible worlds, every one of which is contingent, should become a logically necessary truth. It is strange to think that a set of contingent worlds makes a sentence necessary; that the quantity of worlds decides about the quality of a sentence.

Another objection is that possible worlds have nothing to do with explaining actually used modal concepts. It is natural to think that the actual world transcends present facts. The question is why one should think that what is not happening in the actual world is possible because it is happening in a possible world, instead of thinking that it is possible because it will happen or was possible because it would happen in the actual world. Why should one suppose for any fact, which did not happen but could have happen in the actual world, that there is a possible world in which the fact is happening or has already happened?

The possible worlds semantics explains modal concepts by establishing their truth-conditions. But the truth-conditions are construed in such a way that it is impossible to know whether they are met by any sentence. Lewis seems to have a theory of how to understand modal concepts but, at the same time, the theory excludes the possibility of having criteria to decide whether any sentence is true in a possible world; the theory has no practical consequences.

Still another one follows from the fact that it is disputable whether truthconditions are equivalent to sufficient conditions of meaningfulness. There is a controversy among philosophers about the primary criteria of meaningfulness. Pragmatically oriented philosophers maintain that assertabilityconditions are crucial inasmuch as in some contexts a sentence that is evidently true can nevertheless be classified as nonsense. They say that if not entirely, the sense of a sentence is to a high degree determined by the context in which the sentence is asserted. Logicians seem to ignore this controversy. They would think that a sentence of a decided true-value must be meaningful; even its falsity gives a sufficient reason to call it a possible truth or transform it into an explicit modal sentence. A possible truth cannot be 
nonsense. When, for example, 'It is raining' is false, it is still a possible truth and 'It is possible that it is raining' is always a meaningful sentence because there always exists a possible world in which it is raining. So, the sentence cannot be nonsense. The suggestion is that when people use the operator 'It is possible...' they are never obliged to refer to the actual world but always to a possible world. Criteria of meaningfulness are liberalized to such a degree that a suggestion that a modal sentence, fulfilling the criterion of being true in a possible world, can be nonsense, is a blasphemy.

Similarly, modal logic liberalizes criteria of consistency by introducing the distinction between composite and divided sense. If there are two contradictory sentences $p$ and not- $p$, their conjunction in composite sense 'It is possible that $p$ and not- $p$ ' is self-contradictory, whereas in divided sense ' $P$ and it is possible that not-p' is consistent. So, it seems that contradiction is automatically avoided when two contradictory sentences are asserted in the divided sense ' $P$ and it is possible that not- $p$ '. It is enough that the first one refers to the actual and the second to a possible world to preserve consistency of their conjunction.

Ontological commitments make logicians believe it is unimaginable that epistemology could dictate criteria of meaningfulness for modal contexts. Moore challenges this belief with some pragmatic considerations concerning a set of first-person present-tense sentences. ${ }^{4}$ They are sentences of the following characteristic: Firstly, they include the very special, self-referential indexical term "I". Secondly, they are formulated in the present continuous tense. Thirdly, they concern the qualities of the speaker himself. Fourthly, they are formally contingent. Fifthly, they are analyzed in a pragmatic way, i.e., not as inscriptions but as utterances. Moore's exemplary sentence 'I am standing up' has none of the ontological properties of pseudo-necessary sentences: it is neither inevitable, nor irreversible or unchangeable. On the other hand, Moore credits it with most of the epistemic properties of pseudonecessary sentences: it is ungiveapable, it cannot be rationally rejected and is self-evident.

He thinks that the term "logical possibility" is epistemically indispensable as it serves to define the category of contingent sentence: $p$ is a contingent sentence if it is logically possible that $p$ is true and it is logically possible that not- $p$ is true. Moore interprets this term as a sentential operator 'It is possible that...' and simply undertakes a survey of its pragmatic applications. Namely, he analyses conjunctions of knowledge claims with assertions

${ }^{4}$ Chihara, C. (1998). The Worlds of Possibility, Oxford: Clarendon Press, ss. 76-112. 
of possibility of the type: 'I know that $p$, but it is possible that not-p'. These assertions, as to their form, highly resemble the modal sentences in composite sense ' $P$ and it is possible that not-p' of which it is said that they are unquestionably consistent. If the basic claim of modal ontologists that modal contexts are entirely epistemically independent is justified, then the addition of an epistemic operator should not disturb their meaningfulness, i.e., 'I know that $p$, but it is possible that not-p' should be as meaningful as ' $P$ and it is logically possible that not- $p$ '. Moore intends to argue that, at least in cases of his exemplary sentences, assertions of logical possibility are absurdly false, i.e., senseless, just because they are epistemically dependent.

Moore's prime target is to show that formal contingency, of such a firstperson present-tense sentence as 'I am standing up', does not imply that a person $A$ cannot make with this sentence a safe knowledge claim. He is convinced that he has found counterexamples to the view that contingent facts cannot be known. Importantly, Moore focuses on the fact that in situations where a sentence appears in a safe knowledge claim, from its contingency alone it does not follow that it is possible that the sentence is false.

Moore maintains that his exemplary sentence is epistemically symmetrical. Firstly, while asserting 'I am standing now', a person $A$ always believes that he knows the sentence to be true and, consequently, he is forced to believe that it is then impossible to be false. Secondly, when a person $A$ claims 'I know that I am standing up now', nobody is able to undermine this claim rationally. So, it is sufficient to say 'I am standing up' and the sentence 'It is possible that I am sitting down' becomes always and for everybody false. Ordinary language functions in such a way that a sentence of the type 'It is possible that $p^{\prime}$ cannot be taken to be true when a person $A$, asserting it, is admitted to know that not- $p$. He, who knows that $p$ is false, is forbidden to imply that $p$ is at the same time possibly true. The assertion 'It is possible that $p$ ' is understood as an assertion of ignorance equivalent to 'I don't know that not- $p$ '. Hence, when $A$ claims 'I know that I am standing up', his subsequent assertion 'It is possible that I am sitting down' — suggesting ignorance as to his declared position - should be evaluated as absurdly false: not just false but necessarily false.

Actually, Moore does not make such a strong claim because he too often speaks of implied knowledge instead of knowledge claims. One can only speculate that, if he consistently conjoined explicit knowledge claims and assertions of possibility, their mutual incompatibility, in the case of his firstperson present-tense sentence, would be much more obtrusive. He would then be obliged to say that the assertion 'It is possible that $q$ ', where $q$ is 
a contrary of $p$, is always absurdly false when the knowledge claim 'I know that $p$ ' is safe.

Anyway, Moore holds that the statement "The sentence 'I am standing up' is contingent" does not imply 'It is possible that I am sitting down', whereas 'It is possible that I am sitting down' does imply 'I don't know that I am standing up'. So, in this particular case, the sentence " 'I am standing up' is contingent" does not imply that 'I don't know that I am sitting down' is true. Though 'I am standing up' is contingent, the assertion 'It is possible that I am sitting down' is automatically false, because the knowledge claim 'I know that I am standing up' is safe in all situations.

From the mere fact that $p$ is formally contingent it does not follow that it is possible that $p$ is false. Formal contingency of $p$ is only a necessary condition of possibility that not- $p$, which, to become a sufficient one, must be conjoined with the pragmatic condition of lack of knowledge that $p$.

The term "logically possible" can be taken to be a synonym for "not self-contradictory" - it is then completely free of epistemic implications. Its applications cause misunderstandings when one does not differentiate between two contexts: ' $P$ is logically possible' and 'It is logically possible that $p$ '. Moore suggests that one should decide which context is related to the term "not self-contradictory" by looking into grammar. For him, 'It is not self-contradictory that I am not standing up' is a grammatical nonsense, whereas "The sentence 'I am not standing up' is not self-contradictory" is grammatically allowed. Accordingly, ' $P$ is logically possible' is surely an equivalent of ' $P$ is not self-contradictory'. But it does not mean that ' $P$ is not self-contradictory' and 'It is logically possible that $p$ ' are also equivalents, i.e., when the former sentence is true, the latter is also true. Only if they were equivalents, it would follow from contingency of $p$ that $A$ does not know that $p$.

In other words, ' $P$ is not self-contradictory' entails ' $P$ is logically possible' just because they are meta-linguistic sentences enabling the same classification of object-sentences. By analogy, it seems natural to think that ' $P$ is not self-contradictory' entails 'It is logically possible that $p$ ' - the second one understood as an object-sentence with the operator of possibility. But the last pair consists in fact of different sentences: ' $P$ is not self-contradictory' does not entail 'It is logically possible that $p$ ' because the first if free of and the second one is burdened with epistemic implications.

Moore compares two variants of 'It is logically possible that $p$ ': (1) 'It is logically possible that I could have been sitting down' and (2) 'It is logically possible that I am sitting down'. The first sentence gives a safe context for 
the term "logically possible" in situations where a person $A$ knows that $p$. It would be unnatural for $A$, who knows that he is standing up, to assert the second, whereas it is perfectly natural to assert the first one.

Moore restricts himself to the warning that the sentence 'It is logically possible that not- $p$ ', like 'It is possible that not-p' in ordinary language, can be truly asserted only by a person $A$ not knowing that $p$. Otherwise, though still meaningful, it is false. When $A$, standing up, asserts 'It is logically possible that I am sitting down', he should be understood as implying that he does not know that he is standing up. Moore neglects the question whether such a situation is imaginable, i.e., whether any rational person can admit that he does not know that he is standing up, which would force him to admit that the assertion 'It is logically possible that I am sitting down' is absurdly false.

Importantly, Moore accentuates that from ' $P$ is not self-contradictory' it does not follow that one can safely say 'It is logically possible that $p$ '. A person $A$, who does not know that $p$, can truly say 'It is logically possible that not-p', whereas a person $B$, who knows that $p$, can only falsely say 'It is logically possible that not-p'. His conclusion is meant to concern all formally contingent sentences - he does not consider the option that his exemplary sentences, in virtue of pragmatic analysis, acquire a new status.

From the conjunction of the sentences " 'I am sitting down' is not selfcontradictory" and 'I know that I am not sitting down' it certainly does not follow 'It is logically possible that I am sitting down'. If one can truly assert about oneself 'It is logically possible that I could have been sitting down', then one cannot simultaneously truly say 'It is logically possible that I am sitting down'. The first assertion implies that $A$ knows that he is not sitting down, the second one implies that $A$ does not know that he is not sitting down. These two sentences are incompatible: 'It is logically possible that I could have been sitting down' refers to an unrealized possibility and presupposes that $A$ knows his actual position, whereas 'It is logically possible that I am sitting down' presupposes that $A$ does not know it.

When an epistemologist uses the form 'It is logically possible that $p$ ' in such a way as if it followed from ' $P$ is not self-contradictory', it means that he overlooks the fact that 'It is logically possible that $p$ ', being equivocal, does not automatically imply ' $P$ is not known to be false'. Then, he mistakenly takes 'It is logically possible that $p$ ' as true, even if ' $P$ is known to be false' is true. As a result, he commits the epistemological fallacy of thinking that from 'It is logically possible that not- $p$ ' it must follow ' $P$ is not known'. It is a fallacy because there is a context for logical possibility, which implies 
knowledge and also such which is epistemically neutral. Similarly, a logician is prone to commit the ontological fallacy, when he thinks that all contexts for logical possibility are epistemically neutral.

In the case of Lewis's version of modal realism there is still another story to tell: Moore's sentences do not fit the definition of possible truth in the sense that they can be true only in the actual world. If they are false, they are false only in the actual world and cannot be true in another world. Firstperson present-tense sentence, including two indexical terms "I" and "now", if interpreted ontologically, appears to be boundary sentences for the following reasons. Lewis holds that a speaker establishes actuality. As the speaker himself is at the same time an indispensable part of a world, wherever he exists, he finds himself within the actual world. It is impossible for a speaker to transcend the actual world and appear in another possible world. Moore's sentences, which are self-referential, of necessity say something about the actual world. The assertion 'I am sitting now, but it is logically possible that I am not sitting now' is ontologically absurd because two contradictory facts concerning the speaker cannot happen at the same time in the same world. They could have happened at the same time in different worlds or it the same world at different times. For Moore's sentences both options are excluded. They can describe only actual and present facts and it is not sufficient to formulate a compound sentence in divided sense ' $P$, but it is possible that not- $p$ is the case now' to avoid absurdity. Also 'It is possible that I could have been sitting now' cannot be interpreted as 'There is another world in which I am sitting now' because the speaker cannot find himself in another world. The sentence must be interpreted as concerning the actual world, i.e., as describing a possible truth, but such a one that is true only in the actual world. So, the conjunction 'I am standing up, but it is possible that I could have been sitting now' is consistent because its second part is a possible truth in the actual world which does not contradict the actual truth of its first part. Of course, Moore does not propose ontological analyses of his exemplary sentences simply because Lewis's modal realism was in his times only a possible theory.

For epistemic reasons Moore distinguishes two present-tense applications of the term "logically possible": 'It is logically possible that not-p is the case now' and 'It is logically possible that not- $p$ could have been the case now'. In order not to violate ordinary language the first one must respect the following rule: It is logically possible that not- $p$ is the case now, if $A$ does not know that $p$. The second one respects another rule: It is logically possible that not- $p$ could have been the case now, if $A$ knows that $p$. 
Epistemologists are sometimes unable to realize that the meta-statement 'Not- $p$ is not self-contradictory', which is free of epistemic implications, is not equivalent to the statement 'It is logically possible that not- $p$ ', which, including a modal operator, is epistemically burdened and equivocal. Especially, they do not see that formal contingency of a first-person present-tense sentence is not a sufficient condition for using it meaningfully in the unspecified assertion 'It is logically possible that not-p'. Two basic applications of the operator of logical possibility are determined by the following rule: a person $A$, entitled to say 'It is logically possible that I could not have been standing now', is at the same time banned from saying 'It is logically possible that I am not standing now', because the first statement requires $A^{\prime} s$ knowledge, whereas the second one requires $A^{\prime} s$ lack of knowledge, as to his standing now.

Epistemologists believe that logical possibility is never free of epistemic implications. They interpret "contingency of $p$ " as a sufficient condition of correct assertion of logical possibility that not-p. Ignoring the fact that different assertions of possibility imply incompatible epistemic attitudes, they are tempted to generalize that the meta-statement ' Not- $p$ is logically possible', which is epistemically neutral, implies impossibility of knowledge that $p$. By observing that assertions of the form 'It is logically possible that not-p' require a permanent control over epistemic attitudes towards $p$, in order to chose only one of two incompatible variants of the form, Moore calls this generalization a mistake.

Logicians are prone to make the opposite mistake. They think it is enough to interpret the term "logical possibility" as a quantifier ranging over possible worlds and free the sentence 'It is logically possible that $p$ ' of any epistemic implications. Moore shows that some modal contexts are paradoxical because, formally correct, they are pragmatically absurd. He warns logicians that, at least in some cases, ordinary language stubbornly interprets the phrase "It is logically possible ..." as an epistemic operator. In a sense, ordinary language is unable to ontologize logical possibility unrestrictedly.

Tadeusz Czarnecki

Instytut Ekonomii, Socjologii i Filozofii

Politechnika Krakowska

ul. Warszawska 24

31-155 Kraków 